\title{
IMPACTO DE LA COVID-19 EN EL DESARROLLO ECONÓMICO DE LOS MICRO EMPRESARIOS DE LA CIUDAD DE JIPIJAPA
}

IMPACT OF THE COVID-19 IN THE ECONOMIC
DEVELOPMENT OF THE MICRO ENTREPRENEURS OF
THE CITY OF JIPIJAPA

Garmen Argentina Álvarez Vásquez

Profesora de Emprendimiento e Innovación Tecnológica en la Carrera de Administración de Empresas y

Laboratorio Clínico, (Ecuador).

E-mail: carmen.alvarez@unesum.edu.ec ORCID: https://orcid.org/0000-0002-0099-3853

Jessenia Herminia Morán Chilán

Profesora de microeconomía y macroeconomía en la Carrera de Contabilidad y Auditoría.

Universidad Estatal del Sur de Manabí, (Ecuador).

E-mail: jessenia.moran@unesum.edu.ec ORCID: https://orcid.org/0000-0002-3853-6017

Erick Raúl Baque Sánchez

Máster en Contabilidad y Auditoría, Docente de la carrera de Contabilidad y Auditoría.

Universidad Estatal del Sur de Manabí, (Ecuador).

E-mail: erik.baque@unesum.edu.ec ORCID: https://orcid.org/0000-0002-5223-2474

Recepción: 07/08/2020 Aceptación: 06/11/2020 Publicación: 18/12/2020

\section{Citación sugerida:}

Álvarez, G. A., Morán, J. H., y Baque, E. R. (2020). Impacto de la COVID-19 en el desarrollo económico de los micro empresarios de la ciudad de Jipijapa. $3 C$ Empresa. Investigación y pensamiento crítico. Edición Especial COVID-19: Empresa, China y Geopolítica, 53-65. https://doi.org/10.17993/3cemp.2020. edicionespeciall.53-65 


\section{RESUMEN}

Las microempresas poseen una singular importancia, debido a la capacidad de generación de empleo e ingresos, útiles para que el aparato productivo sea capaz de dinamizar la actividad económica, en Ecuador y en particular en Jipijapa. El impacto de la COVID-19, ha sido devastador en el desarrollo económico de los micro empresarios. La COVID-19, es una enfermedad que ha generado un impacto económico desfavorable. Los sistemas económicos están afectados por esta pandemia y la región latinoamericana, es considerada las más golpeada de todas. En la presente investigación se propone realizar una actualización de los conocimientos, para percibir cuál es la situación que presentan los micro empresarios de la ciudad de Jipijapa, Ecuador, respecto al impacto de la COVID-19, en cuanto a sus planeaciones estratégicas. Para tal fin se desarrolla una investigación a nivel exploratorio, descriptivo y documental, apoyada en una análisis bibliométrico, orientado al análisis de artículos científicos, se presentan resultados de la investigación de campo a 250 microempresarios del cantón Jipijapa, de la provincia de Manabí, en aras de tener una visión real de la problemática que enfrentan los micro empresarios, útil en la concientización de la necesidad de plantear estrategias para su fortalecimiento, innovación y alcance de las principales características que son necesarias para crear ventajas competitivas y posicionarse en el mercado.

\section{PALABRAS CLAVE}

COVID-19, Impacto, Microempresas, Planeaciones estratégicas, Sistemas económicos. 


\section{ABSTRACT}

The micro businesses possess a singular importance, due to the capacitance of generation of employment and revenues, useful in order that the productive apparatus is capable of energize the economic activity, in Ecuador and specially in Fipijapa hat. The impact of the COVID-19, has been devastating in the economic development of the micro entrepreneurs. The COVID-19, it is an illness that has generated an economic unfavorable impact. The economic systems are to be affected for this pandemic and the Latin American region, is considerate the hit more of all. In investigation this letter proposes carry out an update of the knowledge's, to perceive which is the situation they present the micro entrepreneurs of the city of Fipijapa hat, Ecuador, concern to the impact of the COVID-19, as for your strategic planning. For such end an investigation is developed at exploratory, descriptive and documentary level, based one's opinion on a bibliometric analysis, found one's bearings to the analysis of scientific articles, present results of the investigation of field to 250 micro businesses of the Fipijapa hat canton, of the province of Manabi, for the sake of having a real sight of the problematic that meet face to face the managers micro, useful in the awareness of the need to outline strategies for your fortification, innovation and reach of the main characteristics that are necessary to create competitive advantages and position in the market.

\section{KEYWORDS}

COVID-19, Impact, Micro businesses, Strategic planning, Economic systems. 


\section{INTRODUCCIÓN}

Las microempresas son propulsoras de empleo y producción en una sociedad, contribuyen a incrementar el ingreso nacional, ellas son consideradas de importancia social. Requieren ser apoyadas por las diferentes políticas públicas, apropiadas para brindar capacitación permanente en técnicas gerenciales y administrativas, con el fin de proyectar las actividades a desarrollar, así como los resultados esperados, al minimizar el riesgo empresarial y permitir el acceso al financiamiento necesario para fortalecerse.

En Ecuador, los micro empresarios, han recibido apoyo a través de las políticas estatales mediante asistencia técnica y asesoramiento en la creación de microempresas y en el acceso al financiamiento a través de la banca pública (Secretaría Nacional de Planificación y Desarrollo de Ecuador, 2017). El estado ecuatoriano ha ejecutado diversos programas encargados de diagnosticar potencialidades y oportunidades regionales y provinciales, dirigidos al emprendimiento entre empresario - emprendedor, programas capaces de brindar información sobre emprendimiento, asesorías para la puesta en marcha de micro emprendimientos, promoción de la innovación en la empresa y la diversificación productiva, mediante el cofinanciamiento de proyectos innovadores (Salinas et al., 2012).

Estudios de Kantis, Angelelli, y Koening (2004), Chanamé (2008), y Kumar y Bhaduri (2014), confirman que las microempresas son unidades de producción de bienes y servicios, que han demostrado ser un medio efectivo para dinamizar las actividades productivas en la sociedad. Ocupan un lugar sobresaliente tanto en los países en crecimiento como desarrollados.

Las microempresas, consideran el sector informal de forma positiva, al ocupar una distinguida posición en las actividades económicas de las economías en desarrollo y emergentes actuales. Sin embargo, se requiere de una mayor articulación entre los actores públicos y privados, así como la sostenibilidad de los esfuerzos que se realizan para alcanzar un mayor afianzamiento entre las microempresas y los comerciantes informales.

En la zona sur de Manabí, comprendida en los cantones Jipijapa, Paján y Puerto López, existen microempresas dedicadas a la comercialización de productos y servicios. Los micro empresarios se han visto afectados debido al impacto de la COVID-19, en el desarrollo 
económico actual, impacto que ha acarreado indiscutibles repercusiones económicas a nivel mundial.

De acuerdo con el estudio realizado, se constata que las microempresas del cantón Jipijapa, atraviesa una problemática relacionada con la organización interna de sus establecimientos, lo cual afecta la toma de decisiones, por parte de sus administrativos en relación a la información veraz y confiable que deben manejar, lo que disminuye las posibilidades de mayores niveles de rentabilidad y crecimiento sostenido.

Dentro de las deficiencias, de mayor impacto para el cantón Jipijapa, se pueden citar en primera instancia, el ineficiente proceso administrativo, la planeación que busca influir en el futuro al tomar acciones predeterminadas en el presente. Aspectos que requieren de una mayor atención, debido a que la organización requiere de un ordenamiento para hacer que los recursos humanos trabajen unidos en forma efectiva hacia el logro de los objetivos de la empresa.

Se aprecia en el cantón Jipijapa, la escaza capacitación de los microempresarios y del personal que junto a este labora, razón que obstaculiza el aumento de la productividad. Esta situación implica un bajo nivel de competitividad, por otra parte, se aprecian los estilos de comunicación entre los microempresarios y sus subordinados, estilos que imposibilitan que todos los que forman parte de la empresa conozcan los objetivos que se pretenden alcanzar, por lo que el nivel de empoderamiento para trabajar por resultados es limitado.

De acuerdo con lo anterior, se revela que la economía de forma general ha sido golpeada fuerte e indiscriminadamente, según refiere Deloitte (2020), destacada consultora trasnacional. En el caso particular de Ecuador y de acuerdo con el Instituto Nacional de Estadística y Censos del Ecuador (INEC) (2018), en el país existen 899.208 empresas que han sido afectadas, como todas a nivel mundial, razón que ha ocasionado un impacto económico negativo, para el país.

El Observatorio de la Pequeña y Mediana Empresa de la Universidad Andina, refiere que la COVID-19, ha propiciado en el Ecuador, un estancamiento económico que cuesta 900 dólares por cada uno de sus ciudadanos. Ante las actuales condiciones, se estima que la mitad de todas las empresas del país corren el riesgo de quebrar y especialmente, las 
microempresas, que constituyen alrededor del 99\% de todos los negocios en la nación, y son en la actualidad las más afectadas, (Roa, 2020).

Refiere el citado autor, que el segmento de mayor participación en el desenvolvimiento económico ecuatoriano, son las microempresas, las que alcanzan un 91 \%, seguido de las pequeñas empresas, que alcanza un $7 \%$ y las medianas empresas, que alcanzan un 2\%. Datos que se concentran en las áreas de servicio y comercio, los que, según la INEC, generan el 60\%, de todos los puestos de trabajo en Ecuador, (El Universo, 2019; CAMAE - Cámara Marítima del Ecuador, 2020).

Por otra parte, cabe destacar que el impacto que la COVID-19, incide en la planeación estratégica de las pequeñas y medianas empresas (PYMES) ecuatorianas, este impacto ha sido elevado, incluyéndose aquellas referencias que también impactan a la micro y grandes empresas del Ecuador. Esta estimación se hace en vista del amplio espectro de influencia que la COVID-19, ha dejado como evidencia en el ámbito económico en general.

El impacto que la COVID-19, no es comparable a los desastres económicos recientes, por lo que las microempresas, en la actualidad se encuentran desprotegidos, por lo que trabajar de forma colaborativa con empresas e institutos de investigación es fundamental, para realizar estudios y análisis de datos masivos e implementar encuestas que contribuyan a comprender el impacto en las empresas, desde los efectos en ventas e inversión, hasta las complejidades del teletrabajo. Al respecto resulta necesario contar con información que permita tomar las medidas correctas y de manera oportuna, (Cañete, 2020).

Las microempresas, ecuatorianas, tienen la necesidad de modificar la manera de trabajar e incluso, de realizar una total reinvención con fines de enfrentar el impacto económico que la COVID-19, ha ocasionado. Esta situación, mantenida a corto y mediano plazo, requerirá que las empresas deban tener definido un plan de riesgos tanto a nivel estratégico como operacional para hacerle frente.

Las apreciaciones del entorno ecuatoriano y en particular del cantón Jipijapa, referente a los efectos económicos de la COVID-19, en el entorno empresarial, están dadas en los diferentes escenarios relacionados con la actividad empresarial y de negocios a nivel nacional. Cabe destacar que, aunque existen sectores que se han visto mayormente afectadas, es 
importante no perder de vista el hecho de que todo proceso productivo implica una serie de encadenamientos que involucra a múltiples proveedores, incluso de la micro y pequeña industria, a lo largo de las actividades de producción y comercialización.

En Ecuador, no se totalizan, las cifras definitivas respecto al impacto generado por la COVID-19, se conoce que varias empresas se han visto forzadas para realizar ciertos recortes de personal. Por otro lado, a nivel de bancos privados se registró una caída de 3\% en los depósitos captados, como consecuencia inmediata de la paralización de actividades que se dio en la segunda mitad del mes de marzo del 2020, donde se intensificó la presencia de la COVID-19, (Montenegro y Zambrano, 2020).

De acuerdo con lo antes referido, se realiza un análisis basado en el estudio de documentos relacionados con el impacto de la COVID-19, en el desarrollo económico de los micro empresarios de la ciudad de Jipijapa. El análisis se realiza a través de la documentación que sobre el tema existe, para el cual se aplica un estudio bibliométrico, donde se analizan las bases de datos de Scopus y ScienceDirect.

Para complementar la depuración de los documentos relacionados con el impacto de la COVID-19, se realizaron consultas a las bases de datos de ISI Web of Science, Emerald y Jstor. Bases de datos, seleccionadas por criterios de disposición y accesibilidad de la información y por ser referentes ya validados en el desarrollo de análisis bibliométricos.

El análisis bibliométrico es una técnica de investigación validada en múltiples campos como los negocios, nuevas tecnologías, elección pública o la infometría (Wagner et al., 2011). Al respecto, Coombes y Nicholson (2013), Carvalho, Fleury y Lopes (2013), Arduini y Zanfei (2014), y Paiva (2014) resaltan cómo este tipo de análisis permite identificar tendencias en el conocimiento a través de la aplicación de técnicas cuantitativas que enriquecen, entre otras fases de la investigación, la revisión bibliográfica.

También, se depuran los documentos recolectados a partir de la información suministrada por las bases de datos como el año de publicación, palabras clave, campo del conocimiento, tipo y fuente del documento, idioma, instituciones y país. Este procesamiento se realizó mediante el uso de una hoja de cálculo de Excel 2010 del paquete Office. 
El procesamiento de la información se realizó a través del análisis cruzado de contenidos. Este procedimiento, se ejecutó para corroborar la evolución de la COVID-19, en el desarrollo económico de los micro empresarios de la ciudad de Jipijapa.

\section{METODOLOGÍA}

Para el estudio, se tomó una muestra de 250 microempresarios. El análisis documental fue el que se utilizó para constatar el impacto de la COVID-19, en el desarrollo económico de los micro empresarios de la ciudad de Jipijapa.

El análisis se basó en la lógica de la investigación, como en su parte del nivel exploratorio descriptivo, que aprovecha el diseño documental y no-experimental, con el fin de aplicarlo en el contexto objeto de estudio, apoyado en la observación directa y en las encuestas realizadas a los implicados en el proceso. Este proceso tuvo como objetivo realizar una actualización de los conocimientos, para percibir cuál es la situación que presentan los micro empresarios de la ciudad de Jipijapa, Ecuador, respecto al impacto de la COVID-19.

De acuerdo con el enfoque de la investigación propuesta y del análisis de las diversas exploraciones se recuperaron las temáticas relativas al impacto de la COVID-19, en el desarrollo económico de los micro empresarios de la ciudad de Jipijapa, Ecuador. Para tal fin se realizó un análisis bibliométrico donde se analizaron los contenidos, de la temática referida, a través de palabras clave, resúmeness y títulos que permiten identificar el impacto de la COVID-19, en el desarrollo económico de los micro empresarios ecuatorianos.

La bibliometría brinda posibilidades para la recolección, depuración y análisis de documentos científicos que enriquecen la producción de nuevo conocimiento a pesar de las limitantes que su método pueda tener. A través del análisis bibliométrico, se obtiene como aporte al campo del conocimiento, la fundamentación e identificación de los principales factores del impacto de la COVID-19, en el desarrollo económico de los micro empresarios de la ciudad de Jipijapa, Ecuador. 


\section{RESULTADOS}

Del análisis bibliométrico, y de acuerdo con los datos procesados, se identificaron 35 publicaciones en el año 2020, que en líneas generales se adaptaron a los criterios de búsqueda, sin embargo, fue necesario continuar con el proceso de refinamiento de la información, con el propósito de quedar sólo con aquel material que efectivamente sirviera para el análisis de contenidos de acuerdo con los objetivos de la presente investigación. Al respecto se identificó la trascendencia de las microempresas en el entorno económico del país, las que alcanzan un nivel de impacto del $95 \%$, impacto, que ínsita a promover las buenas prácticas, como la planeación estratégica y que es un proceso que otorga el fortalecimiento del control interno de cualquier organización y además optimiza los procesos administrativos y financieros.

En los documentos analizados, se constató que con la presencia de la COVID-19, los micro empresarios y de acuerdo al impacto económico que esta pandemia ocasiona, deben considerar una mayor flexibilidad en sus planes, con el fin de una adaptación a los cambios que surgirán con el paso del tiempo y con el avance de la tecnología. Al respecto este aspecto obtuvo un $85 \%$ de coincidencia, en las publicaciones identificadas a través del análisis bibliométrico.

Otro aspecto que se logró identificar, a través de la documentación obtenida, fue la importancia que tienen las microempresas desde el punto de vista económico en Jipijapa, Ecuador, importancia que reviste una elevada trascendencia social y que ocupa un $87 \%$ de coincidencia al estudiar los documentos obtenidos sobre al impacto de la COVID-19, en el desarrollo económico de los micro empresarios de la ciudad de Jipijapa, Ecuador.

También, se corroboró que con los efectos mencionados se comprende el contexto que en general comparten las microempresas ecuatorianas, ya que, se tiene claro que para comprender el impacto de la COVID-19, en términos económicos, no basta solo con indicar los tantos efectos ocasionados. Se requiere de una concatenación con la investigación y con las experiencias obtenidas a través de los micro empresarios ecuatorianos.

Las necesidades anteriores poseen respuestas, en cuanto a los esfuerzos que se realicen por la formación personal de los micro empresarios, esta actividad es importante para alcanzar prestación adecuada de servicios de alta calidad, minimizar los riesgos de mala praxis y las 
responsabilidades personales y sociales asociadas, en el análisis realizado se constata este aspecto en un $90 \%$.

Las microempresas al ser propulsoras de empleo y producción en una sociedad contribuyen a incrementar el ingreso nacional por lo que son de vital importancia en una sociedad.

El apoyo hacia las microempresas posee un $95 \%$ y el mismo se sustenta a través de las políticas públicas apropiadas, que les permitan capacitación permanente en técnicas gerenciales, administrativas para proyectar las actividades a desarrollar, así como los resultados esperados en aras de minimizar el riesgo empresarial y permitirle el acceso a financiamiento necesario para fortalecerse. Favorecer el crecimiento de las microempresas requiere de inversión en investigación e innovación, con características distintivas, las cuales permitirán la creación de ventajas competitivas a través de la tecnología, con la finalidad de posicionarse en el mercado y de esta manera superar la competencia.

Los resultados descritos y obtenidos a través del análisis documental y con el empleo del análisis bibliométrico se refleja en la Figura 1.

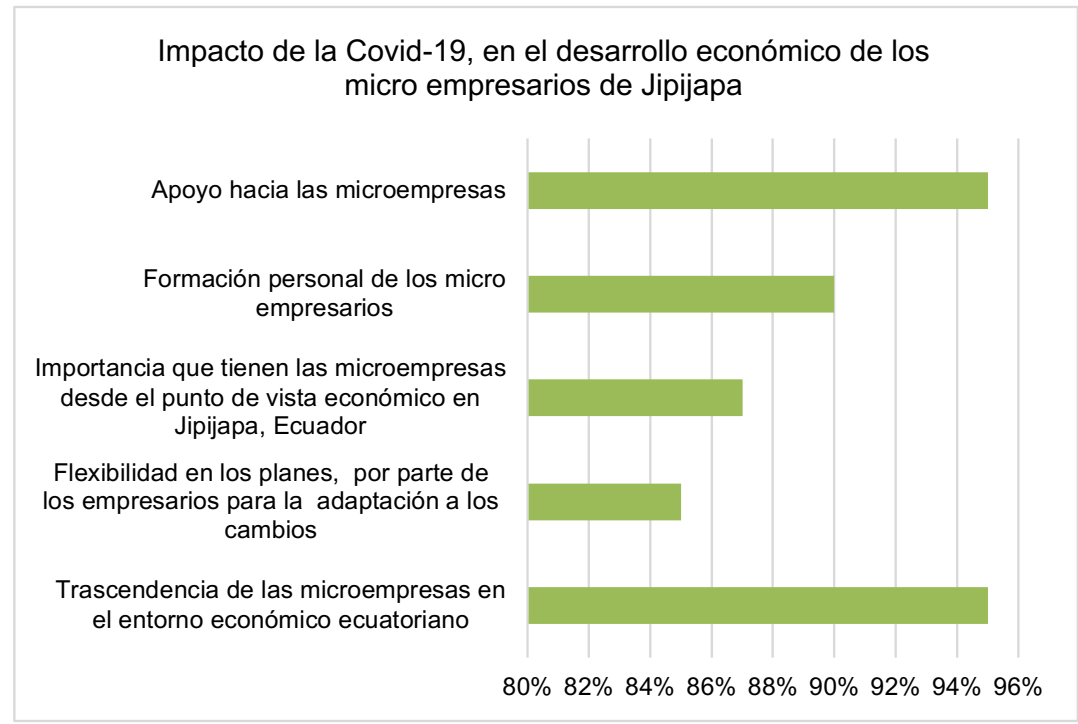

Figura 1. Resultados del análisis bibliométrico sobre el impacto de la COVID-19, en los micro empresarios del cantón Jipijapa.

Fuente: elaboración propia. 


\section{CONCLUSIONES}

De acuerdo, con los resultados y para minimizar el impacto negativo que la COVID-19, ocasiona en las microempresas y propiamente en los micro empresarios, se requiere de la eliminación de los activos y líneas de negocio poco rentables. Se debe identificar los clientes y productos potencialmente que facilitan mayor beneficio, y cuáles ofrecen mayores oportunidades a corto plazo.

También, se debe estimar la incorporación de nueva tecnología o acceder a ella mediante la intervención de un tercero, si se ha conseguido liquidez o el acceso a algún tipo financiamiento. Adecuar o elegir estratégicamente la ruta del negocio a fines de mantenerse en el mercado, constituye un elemento para minimizar los impactos negativos ocasionados por la COVID-19.

De igual forma, se debe actuar con urgencia y replanificar con certeza, las estrategias y tácticas para el nuevo entorno, con planes de acciones concretos, buscar apalancamiento con recursos nuevos, aprovechar las oportunidades del mercado financiero, más en crisis como la del momento. Se requiere de un cuidado especial en el manejo del flujo de efectivo que implica priorizar los gastos para no afectar las proyecciones.

Los factores referidos, provienen, del intercambio de conocimiento o información, relacionada con los micro empresarios, que con sus conocimientos contribuyen al aparato productivo nacional que dinamizan la actividad económica y demuestran su importancia a nivel mundial. Específicamente, los factores incidentes para los micro empresarios, son los relacionados con las deficiencias existentes en las microempresas, causadas por la COVID-19, deficiencias que inciden de forma negativa para realizar la gestión administrativa y desarrollar las actividades a través del cumplimiento de las fases de planeación, organización, dirección, coordinación y control.

\section{REFERENCIAS BIBLIOGRÁFICAS}

Arduini, D., y Zanfei, A. (2014). An overview of scholarly research on public e-services? A meta-analysis of the literatura. Telecommunications Policy, 38(5-6), 476-495. https:// doi.org/10.1016/j.telpol.2013.10.007 
GAMAE - Cámara Marítima del Ecuador. (2020). Banco Mundial aprueba crédito por $\$ 260$ millones para CFN destinado a dar financiamiento a Mipymes. Diario El Universo. http://www.camae.org/economia-del-ecuador/banco-mundial-aprueba-creditopor-260-millones-para-cfn-destinado-a-dar-financiamiento-a-mipymes

Cañete, N. (2020). Coronavirus: ¿Cómo apoyar desde el sector de fomento a la innovación y las pymes? Puntos sobre la i. https://blogs.iadb.org/innovacion/es/innovacion-coronaviruspymes-emprendimientos/

Carvalho, M. M., Fleury, A., y Lopes, A. P. (2013). An overview of the literature on technology roadmapping (TRM): Contributions and trends. Technological Forecasting and Social Change, 80(7), 1418-1437. https://doi.org/10.1016/j.techfore.2012.11.008

Ghanamé, O. (2008). Comentarios a la Constitución. Juristas Editores.

Coombes, P., y Nicholson, J. (2013). Business models and their relationship with marketing: A systematic literature review», Industrial Marketing Management, 42(5), 656664. https://doi.org/10.1016/j.indmarman.2013.05.005

Deloitte. (2020). El impacto económico del COVID-19: los efectos económicos del COVID-19 y los posibles escenarios globales en función de su desarrollo. https://www2.deloitte.com/es/es/ pages/about-deloitte/articles/impacto-economico-del-covid19.html

E1 Universo. (2019, 27 de junio). Las mipymes representan el 99\% de negocios en Ecuador. 
